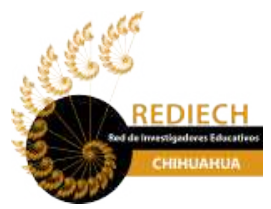

Red de Investigadores Educativos Chihuahua A.C. Chihuahua, México www.rediech.org revista de

de la rediech

ISSN: 2007-4336

ISSN-e: 2448-8550

http://www.rediech.org/ojs/2017/index.php/ie rie rediech/index

Aníbal Zaldívar-Colado

2019

\title{
Laboratorios reales versus laboratorios virtuales en las carreras de ciencias de la computación
}

IE Revista de Investigación Educativa de la REDIECH, 10(18), pp. 9-22. http://dx.doi.org/10.33010/ie_rie_rediech.v10i18.454

\section{(c) $(1)(\theta$}

Esta obra está bajo licencia internacional Creative Commons Reconocimiento-NoComercial 4.0. 


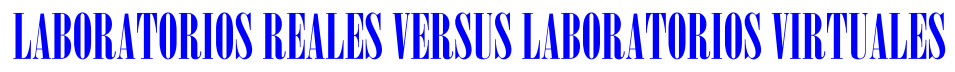

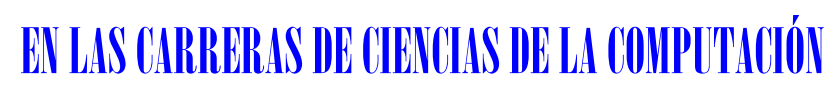

\author{
REAL LABORLITORY IERSIS VIRTILIL LABORITORY
}

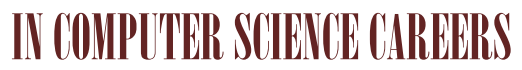

\author{
ZALDÍVAR-COLADO Aníbal
}

Regepción: octubre 22 de 2018 | Aprobado para publicación: febrero 4 de 2019

DOI: https://dx.doi.org/10.33010/ie_rie_rediech.v10i18.454

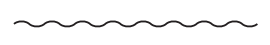

\section{Resumen}

El uso de laboratorios en las carreras de informática y ciencias de la computación es indudablemente valioso para los estudiantes. Gracias a la evolución de las comunicaciones, a través de Internet es posible implementar laboratorios virtuales con costos mucho más bajos que los de uno real, con la ventaja de estar disponible para todos los alumnos a la vez, a cualquier hora y lugar. El presente estudio tiene por objetivo determinar las preferencias de los estudiantes por laboratorios reales y laboratorios virtuales. La investigación es descriptiva correlacional con un enfoque cuantitativo. La técnica de recopilación de datos se realizó con una encuesta aplicada a 483 universitarios mexicanos a través de Internet con la herramienta Google Forms. Entre los principales resultados se obtuvo que el $100 \%$ de los estudiantes encuestados afirma poseer un teléfono inteligente, medio para acceder a los servicios virtuales que ofrece Internet. El 82\% de los sujetos de estudio está satisfecho con los laboratorios físicos, encontrando una preferencia en el uso de laboratorios en línea entre los alumnos que no residen en la localidad donde se ubica su institución educativa, con un coeficiente de correlación $r=0.858$. Se encontraron tendencias en cuanto al tipo de

Aníbal Zaldívar Colado. Profesor-investigador de tiempo completo titular C en la Universidad Autónoma de Sinaloa, México. Es miembro del Sistema Nacional de Investigadores y del Sistema Sinaloense de Investigadores y Tecnólogos. Cuenta con perfil deseable Prodep y es responsable del Cuerpo Académico Señales y Sistemas (UAS-CA-169). Es doctor en educación y maestro en informática por la Universidad Autónoma de Durango. Cultiva la línea de investigación "Tecnología educativa, modelado y simulación de problemas sociales". Director de la Facultad de Informática Mazatlán de la UAS en los periodos 2003-2006 y 2009-2012. Docente de la Facultad de Informática Mazatlán en las asignaturas de matemáticas y redacción de textos científicos. Correo electrónico: azaldivar@uas.edu.mx. ID: http:// orcid.org/0000-0002-6622-6630. 
carrera que cursan los sujetos de estudio; los de ingeniería (ingeniería en sistemas computacionales, ingeniería en sistemas de información, ingeniería mecatrónica e ingeniería de software) se inclinan más al uso de los laboratorios físicos que los alumnos de licenciatura (licenciatura en informática, licenciado en tecnologías de la información y licenciatura en sistemas computacionales). Los universitarios que no residen en la misma ciudad de su institución preferirían el laboratorio virtual. En general, hay una opinión favorable hacia los dos tipos de laboratorios; los estudiantes se sienten satisfechos con su uso y perciben mejoría en las materias de programación y en su rendimiento en general.

Palabras clave: LABORATORIOS, LABORATORIO VIRTUAL, E-LEARNING, EDUCACIÓN SUPERIOR, CIENCIAS DE LA COMPUTACIÓN.

\begin{abstract}
The use of laboratories in computer science and computer science careers is undoubtedly valuable for students. Thanks to the evolution of communications, through the Internet it is possible to implement virtual laboratories with much lower costs than those of a real one, with the advantage of being available to all students at once, from any time and place. This study aims to determine the preferences between real laboratories and virtual laboratories amongst students. The investigation is descriptive correlational with a quantitative approach. The technique of data collection was carried out with a survey applied to 483 Mexican university students through the Internet using the tool Google Forms. Amongst the main results, it was found that $100 \%$ of the surveyed students claim to have a smartphone, which is a means to access the virtual services offered by the Internet. $82 \%$ of the subjects are satisfied with physical laboratories, finding a preference in the use of online laboratories amongst students who do not reside where their educational institution is located, with a correlation coefficient $r=0.858$. Trends were found under the type of degree that subjects belong to, the ones in engineering (computer systems engineering, information systems engineering, mechatronics engineering, and software engineering) are more inclined to the use of physical laboratories than those in computer science, information technology and computer systems. University students who do not reside in the same city as their institution would prefer the virtual laboratory. Overall, there is a favorable opinion towards the two types of laboratories; the students feel satisfied with their use and perceive an improvement in the subjects of programming and in their overall performance.
\end{abstract}

Keywords: LABORATORY, VIRTUAL LABORATORY, E-LEARNING, HIGHER EDUCATION, COMPUTER SCIENCE. 


\section{IITromircuín}

El USO de laboratorios de redes, arquitectura de computadoras, sistemas operativos, bases de datos, ingeniería de software, mecatrónica, etcétera, en las carreras de informática y ciencias de la computación es muy importante para los estudiantes. En ellos se adquiere una visión del área de estudio diferente a la obtenida en el salón de clase tradicional. La llegada de la tecnología a la educación sigue siendo un área escasamente explorada. A pesar de las pocas publicaciones sobre el tema, no hay consciencia sobre la idea de virtualizar y sistematizar los procesos en el marco educativo. Se identifican dos corrientes de pensamiento principales con respecto al empleo de la tecnología con fines de virtualización. La primera versa sobre el apoyo de la operación de la infraestructura de la escuela: optimización de hardware y software mediante el empleo de tecnología de virtualización de servidores, incluidos las soluciones en la nube (Hemanth y Mahammad, 2016). La segunda corriente trata principalmente de tareas específicas dirigidas a la creación y operación de laboratorios virtuales o entornos educativos virtuales (Pizzonia y Rimondini, 2016).

Sobre esta última idea es que se concibe esta investigación, ya que se considera importante el acceso a la infraestructura académica a través de Internet; no solo laboratorios, sino aulas, tutorías, servicios administrativos, etcétera; de ser posible, la integración total de un campus virtual. Ofrecer a los alumnos la posibilidad de realizar trabajos en un laboratorio en línea, pero capaz de resolver las distintas problemáticas que se presentan en el ejercicio real de su área. Se tiene la percepción que motiva pedagógicamente al universitario, lo cual es respaldado por los resultados de investigadores alrededor del mundo (Fiad y Galarza, 2016; Fombona, Pascual-Sevillano y González-Videgaray, 2017; Dyrberg, Treusch y Wiegand, 2017; Pearson y Kudzai, 2015); incluso asignan rasgos lúdicos a estas prácticas (Raessens, 2014; Dippel y Fizek, 2016). La enseñanza de la ciencia, la tecnología y la ingeniería aún están relativamente atrasadas cuando se utilizan nuevos enfoques tecnológicos, especialmente para la educación a distancia en línea. La razón de esta discrepancia radica en el hecho de que estos campos frecuentemente requieren prácticas de laboratorio para proporcionar una adquisición efectiva de habilidades y experiencia real. A menudo es difícil hacer que estos laboratorios sean accesibles a través de Internet. El laboratorio real debe habilitarse para el acceso remoto o debe replicarse como un laboratorio virtual completamente basado en software (Potkonjak et al., 2016). Andersson y Logofatu (2017) aplican una versión modificada de la técnica de aprendizaje cooperativo de rompecabezas (jigsaw) para provocar un cambio y hacer que los estudiantes activamente, y con un alto nivel de responsabilidad, participen en el trabajo de laboratorio basado en la web; los autores consideran, en sus conclusiones, que la motivación juega un papel importante en este cambio.

La motivación es un factor importante para el ser humano al momento de realizar cualquier actividad. En el ámbito educativo es indispensable mantener tanto al estudiante como al docente estimulados y enfocados en el aprendizaje y la enseñanza, respectivamente. En cuanto a los alumnos, la utilización de estrategias innovadoras, de medios y herramientas diferentes a las tradicionales, generalmente funcionan, 
provocando en los discentes ese entusiasmo que los hace ir más allá de lo esperado por el profesor.

Planificar horas de práctica en laboratorios de hardware, software, robótica, telemática, o simplemente asistir a laboratorios de cómputo a realizar tareas, navegar en Internet 0 redactar un trabajo en un procesador de textos motiva más al estudiante que estar sentado en el aula tradicional tratando de asimilar la cátedra del docente. Un problema que rápidamente se percibe es el alto costo económico de construir y equipar adecuadamente un laboratorio como los antes mencionados utilizando software propietario, lo cual podría salvarse con la utilización de software libre. Actualmente, gracias al avance tecnológico de las telecomunicaciones y la simulación de sistemas es posible construir un laboratorio virtual para que los estudiantes de carreras de informática y ciencias de la computación realicen prácticas sobre el desarrollo de software, telemática, robótica, etcétera, con un mínimo de inversión y con la posibilidad de dar acceso a cientos o miles de alumnos a la vez, en cualquier momento y lugar. Podría decirse que la única limitante es la computadora o dispositivo móvil desde el cual se accede al sistema en la web y, obviamente, la capacidad del canal de comunicación (ancho de banda). Esto es, convertir a los laboratorios en línea 0 virtuales en recursos útiles y valiosos en educación superior, especialmente en estudios de ingeniería.

Por lo anterior, esta investigación tiene como principal objetivo determinar las preferencias de los estudiantes, así como las ventajas e inconvenientes, por laboratorios reales, construidos físicamente en una institución educativa, y los laboratorios virtuales, alojados en un servidor y con la posibilidad de acceder a ellos a través de Internet. Durante el proceso del estudio se utilizó el enfoque cuantitativo y la técnica de encuesta para recopilar datos. Los sujetos de investigación son alumnos de educación superior de carreras relacionadas a las ciencias de la computación de diversos estados del país (México).

\section{BSTIIIO DELL IRTE}

Con la finalidad de encontrar estudios relacionados a esta investigación se decidió buscar en bases de datos electrónicas, por lo que se indagó en IEEE Xplore, Elsevier, Springer Link y ACM del área de ingeniería y tecnología; pero también se amplió la exploración al ámbito educativo en Redalyc, Scielo y Scopus. Se realizaron búsquedas en congresos, revistas y libros, incluidos capítulos de libro. Se descartaron los resultados irrelevantes para el tema.

Ushakov et al. (2017) describen un enfoque para crear laboratorios de redes de nube virtual que se utilizan para estudiar tecnologías de red, sistemas operativos y administración de sistemas en instituciones de educación superior. Se desplegó un pequeño centro de datos para llevar a cabo la investigación experimental. Los resultados del experimento muestran la eficiencia del uso de sistemas en la nube en la educación de tecnologías de la información (TI). Andersson y Logofatu (2017) discuten el uso de la técnica del rompecabezas (jigsaw) en clases de laboratorio de 12 e-learning para estudiantes de pregrado de ingeniería mecánica, la cual se lleva a 
cabo en la modalidad blended learning, por lo que los autores consideran la deserción escolar, pues, según afirman, un problema común en la enseñanza de cursos basados en e-Learning es el alto riesgo de que los estudiantes que inician el curso abandonen el programa.

Di Giamberardino y Temperini (2017), en su investigación "Adaptive access to robotic learning experiences in a remote laboratory setting", describen la arquitectura de un pequeño laboratorio remoto disponible mediante un sistema adaptativo basado en web (Mindlab2). El sistema admite experiencias prácticas de e-learning con robótica y automatización realizadas a través de Internet.

El diseño de un laboratorio fotovoltaico remoto es descrito en Axaopoulos et al. (2017), afirmándose que pueden ser herramientas educativas muy útiles, lo que permite la experimentación utilizando equipos reales y funcionales a distancia. Una de las mayores ventajas que posee un sistema de este tipo es la posibilidad de acceder desde cualquier lugar del mundo en cualquier momento del día, siempre que haya una conexión a Internet disponible.

Wuttke, Hamann y Henke (2015) describen un nuevo enfoque holístico para integrar laboratorios remotos y virtuales en el proceso educativo. El objetivo de la integración es evaluar el conocimiento de los estudiantes, no solo en niveles más bajos, como las pruebas de opción múltiple y actividades similares, sino en habilidades de pensamiento de orden superior. Sobre la base de estas pruebas, los alumnos deben obtener una retroalimentación detallada acerca de sus fortalezas, debilidades o concepciones erróneas. Por lo tanto, los métodos de learning analytics se aplican a una base de datos, almacenando las actividades de aprendizaje de los estudiantes durante su fase de trabajo en línea en el proceso educativo. El documento analiza el enfoque real, así como la arquitectura de la implementación de un prototipo.

Ruano et al. (2016) presentan una metodología para crear laboratorios eficaces que interactúan con un ambiente virtual de aprendizaje para lograr una integración avanzada. Se basa en aspectos pedagógicos y considera no solo la aplicación del laboratorio en sí misma, sino también los recursos relacionados que lo complementan. La metodología es flexible, cubre todos los casos posibles y está estructurada en etapas que se pueden usar con cualquier arquitectura de sistema, estándares 0 tipo de laboratorio en línea (virtual, remoto o híbrido), ya que abstrae los aspectos técnicos a un alto nivel.

El Club de Robótica de la Escuela de Ingeniería de la Universidad de Buenos Aires es un espacio donde afrontan proyectos que los incentiven y les muestre que estudiar ingeniería no tiene por qué ser aburrido; es una actividad extracurricular que tiene como objetivo ser un espacio para el aprendizaje de la robótica. Además, describen la organización y la metodología de trabajo del club (Chiesa et al., 2013).

Debido a que los hábitos de aprendizaje de los estudiantes están evolucionando y los cursos teóricos no son atractivos, el trabajo en laboratorios es mucho más motivante. Sin embargo, para realizar actividades específicas, como las de metrología, requieren un costoso laboratorio equipado. Para llevar a cabo experimentos prácticos reales, Ballu et al. (2016) están diseñando un laboratorio virtual. Se integra en Moodle como una nueva actividad para establecer un vínculo con otras más (cursos, exámenes, etcétera) y para garantizar el seguimiento de los alumnos. Un primer prototipo del 
laboratorio virtual está dedicado a la metrología dimensional y geométrica con dispositivos de medición tradicionales simulados (manómetro, micrómetro, indicador de cuadrante, entre otros) y máquinas de medición de coordenadas. La simulación de mediciones se realiza en un entorno tridimensional y se basa en modelos de piezas con dimensiones, orientación, errores de posición y forma (formas de modelos de piel) y en modelos de dispositivos de medición con incertidumbres de medición.

Los laboratorios virtuales y las simulaciones interactivas son excelentes enfoques para capacitar a los estudiantes en principios técnicos, lo que puede ser útil en muchos campos de la enseñanza de la ciencia y la ingeniería. Para este propósito, Redel-Macías et al. (2016) han desarrollado un entorno virtual que puede simular operaciones reales de laboratorio, mejorando efectivamente el proceso de enseñanza con una interfaz intuitiva y atractiva. Dicho software muestra la caracterización de la práctica de estudio virtual de las propiedades básicas de los biocombustibles, simulando la realidad paso a paso. Este laboratorio virtual ha sido utilizado por estudiantes de la maestría en biomasa para generación de energía. El objetivo de esta herramienta es ayudar a los alumnos a estudiar, aprender e investigar por su cuenta. El laboratorio virtual está hecho de una aplicación basada en la web para complementar la capacitación de laboratorio experimental, permitiendo a los discentes preparar sus prácticas experimentales antes de ir al laboratorio y revisarlas en cualquier momento. La aplicación exhibe características educativas clave de laboratorio virtual como un diseño integrador y pruebas de autoevaluación. Permite un proceso de aprendizaje activo y personalizado, adaptabilidad a los objetivos del profesor y versatilidad y simplicidad utilizando diferentes recursos multimedia. Dicha herramienta fue evaluada positivamente por los estudiantes; la mayoría consideró que promovió el aprendizaje y el esfuerzo personal, siendo una excelente herramienta de preparación para experimentos reales.

Se muestra la experimentación remota utilizando tecnología móvil con fines didácticos en Granado et al. (2013), donde se afirma que los estudiantes que usan dispositivos móviles, como teléfonos inteligentes o tabletas, pueden interactuar con hardware de laboratorio físico a través de una interfaz de dispositivo móvil; el usuario puede verificar en tiempo real lo que sucede en el hardware del laboratorio cuando cambian los parámetros del sistema.

En la India, el proyecto Virtual Labs se ha constituido como un esfuerzo nacional para proporcionar acceso a Internet y laboratorios virtuales basados en navegador a estudiantes universitarios de ciencias e ingeniería. Actualmente hay más de 800 experimentos en 70 Virtual Labs de más de diez disciplinas de ciencia e ingeniería. Se han realizado más de quinientos talleres sobre estos laboratorios virtuales y los experimentos se han utilizado más de dos millones de veces. Virtual Labs es una importante iniciativa del gobierno para la educación superior en ingeniería en la India. Debido a lo anterior, Kumar, Emory y Chopella (2018) examinan los laboratorios virtuales desde una perspectiva de usabilidad, centrándose en Virtual Labs, considerado los elementos de la interfaz de usuario (usabilidad técnica), así como la forma en que ayudan al aprendizaje (usabilidad pedagógica); también adaptando la heurística de usabilidad técnica de Nielsen y los criterios de usabilidad pedagógica 14 de Nokelainen a laboratorios virtuales y evaluaron la usabilidad de los tres Virtual 
Labs más utilizados; pero, además, construyendo listas de verificación para cada uno de estos criterios y evaluando los Virtual Labs en contra de las listas.

El desarrollo de un laboratorio b-learning (blended learning) experimental para un curso de pregrado de máquinas eléctricas es presentado en Pérez Pinal et al. (2016), para lo cual se desarrolló un tablero de señales de acondicionamiento personalizado que se adapta a una placa de adquisición de datos utilizada para interactuar con el objetivo de tiempo real de LabVIEW. Además, se realizó una interfaz gráfica de usuario que permite la interacción remota en tiempo real.

De acuerdo a lo observado en esta sección, se confirma la utilidad y capacidad pedagógica de los laboratorios reales (físicos) y virtuales en los estudios de ingeniería, donde están inmersas las ciencias de la computación.

\section{Netrounouaril}

Durante el proceso, la investigación se basó en el enfoque cuantitativo, definido por Arias (2012) como el utilizado cuando el objetivo es describir ciertas características de un grupo mediante la aplicación de un cuestionario. El análisis estadístico más elemental radica en la elaboración de una tabla de distribución de frecuencias absolutas y relativas o porcentajes, para luego generar un gráfico a partir de dicha tabla. Por su nivel es una investigación explicativa; es decir, está dirigida a responder las causas de los eventos y fenómenos físicos o sociales. Como su nombre lo indica, su interés se centra en explicar por qué ocurre un fenómeno y en qué condiciones se manifiesta o por qué se relacionan dos o más variables (Sampieri, Fernández y Baptista, 2014). Por su diseño, es un estudio de campo, por lo que consiste en la recolección de datos directamente de los sujetos investigados o de la realidad donde ocurren los hechos (datos primarios), sin manipular o controlar variable alguna; es decir, el investigador obtiene la información, pero no altera las condiciones existentes. De allí su carácter de investigación no experimental (Arias, 2012).

Se determinó que la población la conforman la totalidad de los estudiantes de educación superior del país (México) inscritos en programas de informática, ciencias de la computación y similares que hagan uso de laboratorios reales y/o virtuales. Para la obtención de datos primarios se diseñó una encuesta estructurada con 33 ítems divididos en tres categorías (datos personales, tecnología y laboratorios), autocumplimentada por el alumno, garantizando el anonimato. En el instrumento se les cuestionaba a los discentes sobre sus preferencias acerca de los laboratorios, tanto físicos como en línea, así como por las ventajas y desventajas que perciben entre las dos modalidades. La encuesta se implementó en la web con Google Forms y se distribuyó por correo electrónico y redes sociales (WhatsApp y Facebook) a los participantes. A pesar de distribuir la encuesta en la mayoría de las entidades mexicanas, únicamente respondieron universitarios de los estados de Sinaloa, Sonora, Baja California, Nayarit, Colima, Ciudad de México, Puebla, Estado de México e Hidalgo, respondiendo en mayor cantidad sujetos de Sinaloa. En total respondieron 483 estudiantes de educación superior. 


\section{Resilitinos}

En esta sección se analizan e interpretan los resultados obtenidos de la encuesta. Como ya se dijo, el instrumento se envió a estudiantes y docentes (para que lo distribuyeran a sus alumnos) de 25 estados de la República Mexicana, respondiendo 483 sujetos de nueve entidades, 230 mujeres y 253 hombres, sin observarse un sesgo importante por el género del encuestado. De ellos, 135 sujetos de estudio cursan las carreras de licenciatura en informática, licenciado en tecnologías de la información y licenciatura en sistemas computacionales; 348 pertenecen a ingeniería en sistemas computacionales, ingeniería en sistemas de información, ingeniería mecatrónica e ingeniería de software. En la tabla 1 se ilustra la distribución por género y edad de los discentes que respondieron al instrumento; en la última columna y última fila se muestran las cifras totales en cada categoría.

\begin{tabular}{cccc}
\hline \multicolumn{4}{c}{ Tabla 1. Distribución por género y edad } \\
\hline Edad & Mujeres & Hombres & Total \\
\hline 17 & 33 & 38 & 71 \\
\hline 18 & 32 & 31 & 63 \\
\hline 19 & 19 & 29 & 48 \\
\hline 20 & 34 & 33 & 67 \\
\hline 21 & 36 & 29 & 65 \\
\hline 22 & 18 & 30 & 48 \\
\hline 23 & 30 & 28 & 58 \\
\hline 24 & 28 & 35 & 63 \\
\hline Total & $\mathbf{2 3 0}$ & $\mathbf{2 5 3}$ & $\mathbf{4 8 3}$ \\
\hline Fuente: Elaboración propia. & & \\
\hline
\end{tabular}

Como se observa en la tabla 1, no se percibe un sesgo claro entre los totales por género, 230 y 253 , respectivamente. Tampoco por edades se presentan diferencias significativas, salvo en los estudiantes de 19 años, donde 19 son mujeres y 29 hombres; y los de 22 años, que agrupa a 18 del sexo femenino y 30 del masculino.

De acuerdo con el lugar donde estudian se muestran las distribuciones en la tabla 2.

\begin{tabular}{lccccccccc}
\hline \multicolumn{1}{c}{ Tabla 2. Distribución por estado } \\
\hline & Sin & Son & BC & Nay & Col & CDMX & Pue & EMex & Hid \\
\hline F & 113 & 18 & 17 & 33 & 21 & 6 & 8 & 7 & 7 \\
\hline M & 127 & 22 & 20 & 32 & 19 & 9 & 10 & 6 & 8 \\
\hline T & $\mathbf{2 4 0}$ & $\mathbf{4 0}$ & $\mathbf{3 7}$ & $\mathbf{6 5}$ & $\mathbf{4 0}$ & $\mathbf{1 5}$ & $\mathbf{1 8}$ & $\mathbf{1 3}$ & $\mathbf{1 5}$ \\
\hline Sin: Sinaloa. Son: Sonora. BC: Baja California. Nay: Nayarit. Col: Colima. \\
CDMX: Ciudad de México. Pue: Puebla. EMex: Estado de México. Hid: Hidalgo. \\
Fuente: Elaboración propia.
\end{tabular}

La tabla 2 contiene la distribución de los sujetos de estudio por entidad y género. El estado de Sinaloa presenta la mayor frecuencia, siendo el lugar de adscripción de los investigadores; le sigue Nayarit con 65, Sonora y Colima con 40 cada uno, Baja California con 37 (la quinta entidad que más sujetos aportó a la investigación), 
Puebla con 18, Ciudad de México e Hidalgo 15 cada uno y Estado de México con 13. Es importante resaltar que también se les preguntó si residen en la misma población donde se ubica su institución; aunque todos lo hacen en la misma entidad donde estudian, algunos no habitan donde se ubica su escuela.

Referente a la carrera que estudian, 348 son estudiantes de ingeniería (de carreras relacionadas a la informática y computación) y 135 estudian una licenciatura, también del área de la computación. Entre las mujeres, 61 son estudiantes de licenciatura y 169 de ingeniería. Del sexo masculino, 74 de licenciatura y 179 de ingeniería.

Entre los alumnos encuestados, 65 afirman trabajar de tiempo completo y 418 dicen no hacerlo de jornada completa, siendo muy pocos lo que laboran por horas. Es de resaltar que únicamente el 13\% labora ocho horas por día.

En cuando al uso de tecnologías, se les preguntó si tienen teléfono inteligente (smartphone), tableta, computadora y conexión a Internet. Las respuestas a estas interrogantes se muestran en la tabla 3.

\begin{tabular}{lcc}
\hline \multicolumn{3}{c}{ Tabla 3. Acceso a tecnología } \\
\hline & Sí & No \\
\hline Smartphone & 483 & 0 \\
\hline Tableta & 69 & 414 \\
\hline Computadora & 69 & 414 \\
\hline Internet & 419 & 64 \\
\hline
\end{tabular}

Fuente: Elaboración propia.

Como se ilustra en la tabla 3, todos los universitarios encuestados afirman poseer un teléfono inteligente, pero únicamente 69 aseguraron tener una tableta y el mismo número respondió tener una computadora (no necesariamente los mismo 69 sujetos de estudio). En contraste, 419 dicen contar con conexión a Internet en su lugar de residencia.

Acerca del uso de los laboratorios reales (físicos), 81 estudiantes dicen utilizarlos de 0 a 4 horas por semana; 246, de 5 a 6 horas semanales; 30 de 7 a 10 horas y 126 aseguran que asisten a ellos más de 10 horas semanales. Los horarios de uso son comúnmente por la tarde, como lo demuestra la respuesta a en qué horario prefiere asistir al laboratorio; 412 respondió que en horario vespertino y solo 71 dijo que en el matutino.

Respecto al laboratorio virtual (en línea), 80 respondieron que prefieren utilizarlo por la mañana, 140 por la tarde y 263 por la noche. En cuanto a las horas de uso, 69 dicen acceder a él de 0 a 4 horas por semana, 276 lo hacen de 5 a 8 horas, 19 de 9 a 12 horas y 119 aseguran conectarse más de 12 horas.

La siguiente sección de la encuesta contempla preguntas relacionadas a la satisfacción que percibe el estudiante sobre los laboratorios tanto reales como virtuales. Los primeros 11 ítems de este apartado son tipo Likert en una escala que va de uno hasta seis: 1 para "No aplica", 2 "Completamente en desacuerdo", 3 "En desacuerdo", 4 "Ni de acuerdo ni en desacuerdo (neutral)", 5 "De acuerdo" y 6 "Completamente de acuerdo". Se aclara que a las opciones 1, 2 y 3 no respondió ni uno solo de los alumnos. Un resumen a estas 11 afirmaciones se muestra en la tabla 4. 


\begin{tabular}{lccc}
\hline \multicolumn{4}{c}{ Tabla 4. Satisfacción de los laboratorios } \\
\hline Afirmación & $\mathbf{4}$ & $\mathbf{5}$ & $\mathbf{6}$ \\
\hline Estoy satisfecho con el LR & 85 & 260 & 138 \\
\hline Ningún aspecto del LR debe mejorarse & 89 & 258 & 136 \\
\hline Estoy satisfecho con el LV & 87 & 271 & 125 \\
\hline Ningún aspecto del LV debe mejorarse & 81 & 277 & 125 \\
\hline Me desempeño muy bien en el LR & 100 & 250 & 133 \\
\hline Me desempeño muy bien en el LV & 80 & 269 & 134 \\
\hline Estoy satisfecho con el profesor del LR & 99 & 267 & 117 \\
\hline Estoy satisfecho con el soporte del LV & 90 & 260 & 133 \\
\hline El LV debe utilizarse como complemento al LR & 78 & 274 & 131 \\
\hline Considero que el LR mejora mi rendimiento & 74 & 267 & 142 \\
\hline Considero que el LV mejora mi rendimiento & 86 & 266 & 131 \\
\hline LR
\end{tabular}

LR: laboratorio real. LV: laboratorio virtual. 4: neutral. 5: de acuerdo. 6: completamente de acuerdo.

Fuente: Elaboración propia.

De acuerdo con las respuestas en la tabla 4 se observa que, como se dijo anteriormente, ninguno de los estudiantes respondió a "No aplica", "Completamente en desacuerdo" o "En desacuerdo"; las únicas opciones que consideraron los sujetos del estudio son para "Neutral", "De acuerdo" y "Completamente de acuerdo". Se analizan enseguida las respuestas a estos ítems.

A la afirmación "Estoy satisfecho con el LR", 85 alumnos respondieron ser neutrales al respecto, correspondiendo a un 17.60\%. 260 dicen estar "De acuerdo" (53.83\%) y 138 están "Completamente de acuerdo", el 28.57\%. El siguiente es "Ningún aspecto del LR debe mejorarse", a lo que respondieron: "Neutral", 89 (18.43\%); "De acuerdo", 258 (53.42\%); "Completamente de acuerdo", 136 (28.16\%).

A la afirmación "Estoy satisfecho con el LV", las respuestas fueron 87 (18.01\%), $271(56.11 \%)$ y $125(25.88 \%)$, para "Neutral", "De acuerdo" y "Completamente de acuerdo", respectivamente. Para el ítem "Ningún aspecto del LV debe mejorarse" se muestran los porcentajes de las respuestas en el mismo orden anterior; las frecuencias se ilustran en la tabla 4, 16.77\%, 57.35\% y 25.88\%, para "Neutral", "De acuerdo" y "Completamente de acuerdo", respectivamente. Además, el 20.70\% de los encuestados afirman ser neutrales respecto a "Me desempeño muy bien en el LR", el 51.76\% dice estar "De acuerdo" y 27.54\% "Completamente de acuerdo". Sobre el laboratorio en línea, se les planteó el ítem "Me desempeño muy bien en el LV"; los universitarios respondieron 16.56\%, 55.69\% y 27.74\% "Neutral", "De acuerdo" y "Completamente de acuerdo", respectivamente.

También se les presentaron las afirmaciones "Estoy satisfecho con el profesor del LR" y "Estoy satisfecho con el soporte del LV"; a la primera respondieron ser neutrales el 20.50\% de los encuestados, 55.28\% dijo "Estar de acuerdo" y 24.22\% estar "Completamente de acuerdo". A la segunda afirmación respondieron de la siguiente manera: $18.63 \%$ neutrales, $53.83 \%$ "De acuerdo" y $27.54 \%$ "Completamente de acuerdo".

El 83.85\% dijo estar de acuerdo o completamente de acuerdo en que el laboratorio virtual debe utilizarse como complemento del laboratorio físico; $16.15 \%$ se mostró neutral al respecto. Además, el $84.68 \%$ afirma estar de acuerdo o completamente 
de acuerdo en que el laboratorio real mejora su rendimiento académico; un 15.32\% se dice neutral a esta postura. Por último, el $82.19 \%$ considera que el laboratorio en línea mejora su desempeño en la escuela; este porcentaje es la respuesta combinada a "De acuerdo" y "Completamente de acuerdo"; solo el 17.81\% no está de acuerdo ni en desacuerdo.

En la última parte de la encuesta se les cuestionó respecto a en qué materia perciben una mejoría debido a las prácticas en los laboratorios tanto físicos como virtuales; las respuestas fueron similares en el uso de las dos modalidades: 66 afirman haber mejorado en la materia de matemáticas gracias al laboratorio real y 62 dicen mejorar en la misma asignatura gracias al laboratorio virtual. También, 417 aseguran mejorar en las materias del área de programación por las prácticas que realizan en el laboratorio físico y 421 creen que su mejoría en programación se debe al uso del laboratorio en línea.

Entre los alumnos, 257 (53.2\%) optaría por el laboratorio real de tener que elegir entre uno de los dos y 226 (46.8\%) adoptarían el virtual. Además, el $86.3 \%, 417$ estudiantes, quisieran que el laboratorio virtual estuviera integrado a redes sociales; el 13.7\%, 66 discentes, prefiere que no se incorpore el laboratorio en línea a alguna red social.

Para determinar si hay relación entre las variables utilizadas en este estudio se empleó el coeficiente de correlación de Pearson $(r)$, que sirve para determinar el grado de asociación lineal entre dos variables; esta medida oscila entre -1 y +1 . Un valor de $r$ cercano a cero implica poca o ninguna influencia entre las dos variables. En cambio, si está próximo a 1 significa una alta relación positiva, donde el incremento en los valores de una variable influye incrementando a la otra. Un coeficiente de correlación en la frontera de -1 indica una relación negativa entre las dos variables, impactando el aumento de una en la disminución de los valores de la otra; un valor de $r=1$ sería una perfecta relación entre dos variables, lo cual no sucede al modelar problemas reales; al igual tampoco se encuentra $r=0$. Debido a lo anterior, los valores cercanos a 1 son significativos, pero próximos a cero indican que existe poca relación lineal. Este método estadístico ha sido utilizado ampliamente en la investigación educativa en todos los niveles, como lo indican los trabajos de Roux y Anzures, 2014; Heredia y García, 2013; García, Fonseca y Concha, 2015.

Apoyado en lo anterior, se encontraron relaciones relevantes entre las variables lugar de residencia y horas de uso del laboratorio virtual con un $r=0.858$. También es significativo el grado de asociación entre el tipo de carrera, ingeniería o licenciatura y horas de uso del laboratorio físico con $r=0.735$. Aunque no con valores altos como los anteriores, se percibe una relación entre el lugar de residencia y la pregunta "¿De tener únicamente un laboratorio cuál preferirías?" con un coeficiente $r=0.504$. No se encontraron otras correlaciones significativas entre el resto de las variables. Se hace mención aquí a la interpretación que propone Díaz (2017), quien indica que si $\pm 0.40 \leq r< \pm 0.70$ existe una correlación significativa y si $\pm 0.70 \leq r< \pm 1.00$ existe un alto grado de correlación. 


\section{Discissión y concullisiones}

De acuerdo con el objetivo de la investigación, determinar las preferencias de los estudiantes, así como las ventajas e inconvenientes, por laboratorios reales y los virtuales, se encontró que tanto los universitarios que cursan carreras de ingeniería como los de licenciaturas del área de las ciencias de la computación se inclinan por el uso de laboratorios en las dos modalidades, con una tendencia hacia el uso de los virtuales por aquellos que residen fuera de la población que aloja a su institución educativa. Granado et al. (2013) describen en su investigación el uso de laboratorios virtuales como apoyo al aprendizaje y afirman que proporciona a los estudiantes una herramienta para aprovechar su tiempo libre en la universidad. Los discentes pueden realizar las prácticas de laboratorio incluso cuando las instalaciones están cerradas. Todas las actividades de los usuarios se registran en una base de datos para su posterior revisión por parte del profesor. Además, los alumnos pueden almacenar sus experimentos para hacer un análisis de resultados fuera de línea. Lo anterior contrasta con la encontrado en esta investigación, donde en su mayoría los universitarios foráneos afirman utilizar el laboratorio en línea de 9 a 12 horas por semana o hasta más de 12, contrario a lo que respondieron quienes viven cerca de su escuela, que seleccionaron 0 a 3 o 4 a 8 horas de uso semanales. Además, el $82 \%$ de los estudiantes encuestados afirman estar satisfechos con el laboratorio real y el mismo porcentaje responde igual respecto al virtual. Los alumnos no consideran que deba mejorarse ninguno de sus aspectos. El 79\% de los encuestados cree desempeñarse perfectamente en el laboratorio físico y el 83\% percibe un buen desempeño en el laboratorio en línea. La mayoría asegura que los laboratorios reales o virtuales los han hecho mejorar en las materias de programación. Adicionalmente, del $82 \%$ de los sujetos de estudio afirma que el uso de los laboratorios ha influido positivamente en su rendimiento.

Respecto a las posibles diferencias por el tipo de carrera, los estudiantes de ingeniería (en sistemas computacionales, en sistemas de información, mecatrónica e ingeniería de software) se inclinan más al uso de los laboratorios físicos que los alumnos de licenciaturas (en informática, en tecnologías de la información y en sistemas computacionales). Los universitarios que no residen en la ubicación de su institución afirman que de tener que elegir una de las dos opciones preferirían el laboratorio virtual.

Se tiene una opinión favorable, en general, por el uso de los laboratorios físicos o en línea; los estudiantes se sienten satisfechos con su uso y perciben mejoría en las materias de programación y en su rendimiento en general. Con respecto a las ventajas 0 inconvenientes que encuentran los estudiantes en el uso de los laboratorios, dicen no encontrar inconvenientes o puntos de mejora, pero los alumnos foráneos prefieren los laboratorios virtuales, lo cual es una ventaja para quienes residen fuera de la población donde se ubica su centro de estudios, teniendo la posibilidad de realizar prácticas a través de Internet mediante los laboratorios virtuales, optimizando el tiempo y disminuyendo costos de traslado.

Los resultados obtenidos serán de utilidad a los grupos de investigación del área de ingeniería y tecnología de la Universidad Autónoma de Sinaloa, especialmente 20 a los cuerpos académicos de la Facultad de Informática Mazatlán de la mencionada 
institución. Además, se espera sirvan como insumo para la actualización y reforma de los planes y programas de estudio que realiza el Colegio de Ingeniería y Tecnología del mismo centro educativo.

\section{ReFrererills}

Andersson, C. y Logofatu, D. (2017). Using a modified jigsaw technique in e-Learning Laboratory classes for engineering students. En F.L. Wang, O. Au, K.K. Ng, J. Shang y R. Kwang (eds.). International Symposium on Educational Technology. https://dx.doi.org/10.1109/ ISET.2017.62

Arias, F. (2012). El proyecto de investigación (6a. ed.). Venezuela. Editorial Episteme.

Axaopoulos, P.J., Fylladitakis, E.D., Theodoridis, M. y Moutsopoulos, K. (2017). e-Learning applications for remotely accessible photovoltaic array educational laboratories. En C. Douligeris y M.E. Auer, (eds.), IEEE Global Engineering Education Conference (EDUCON).

Ballu, A., Yan, X., Blanchard, A., Clet, T., Mouton, S. y Niandou, H. (2016). Virtual metrology laboratory for e-Learning. Procedia CIRP, 43(2016), 148-153.

Chiesa, A.L., Corbellini, E.M., González, T.A. y Burman, A. (2013). A robotics club experience as a complement to engineering education. IEEE Latin America Transactions, 11(1), 585-590.

di Giamberardino, P. y Temperini, M. (2017). Adaptive access to robotic learning experiences in a remote laboratory setting. Popescu, D., Șendrescu, D., Roman, M., Popescu, E. y Bărbulescu, L. 18th International Carpathian Control Conference (ICCC). Llevado a cabo en Sinaia, Rumania.

Díaz, J.E. (2017). Correlación y regresión lineal de la evaluación tiempo y puntaje con recurso interactivo flash. INNOVA Research Journal, 2(10), 1-8. Recuperado de http://www. journaluidegye.com/magazine/index.php/innova/article/view/254/461

Dippel, A. y Fizek, S. (2016). Playful laboratories the significance of games for knowledge production in the digital age. En CfP: DiGRA 2015 -Diversity of play: Games - Cultures Identities. Llevado a cabo en Lüneburg, Germany.

Dyrberg, N.R., Treusch, A.H. y Wiegand, C. (2017). Virtual laboratories in science education: Students' motivation and experiences in two tertiary biology courses. Journal of Biological Education, 51(4), 358-374.

Fiad, S.B. y Galarza, O.D. (2015). El laboratorio virtual como estrategia para el proceso de enseñanza-aprendizaje del concepto de mol. Formación Universitaria, 8(4), 3-14. https:// dx.doi.org/10.4067/S0718-50062015000400002

Fombona, J., Pascual-Sevillano, M.A. y González-Videgaray, M.C. (2017). M-learning and augmented reality: A review of the scientific literature on the WoS Repository. Comunicar, 25(52).

García, F., Fonseca, G. y Concha, L. (2015). Aprendizaje y rendimiento académico en educación superior: un estudio comparado. Revista Electrónica Actualidades Investigativas en Educación, 15(3). https://dx.doi.org/10.15517/aie.v15i3.21072

Granado, E., Colmenares, W., Pérez, O. y Cataldo, G. (2013). Remote experimentation using mobile technology. IEEE Latin America Transactions, 11(4), 1121-1126.

Hemanth, G.S. y Mahammad, S.N. (2016). An efficient virtualization server infrastructure for e-schools of India. En Satapathy, S.C., Mandal, J.K., Udgata, S.K., Bhateja, V. (eds.). 3er International Conference on Information Systems Design and Intelligent Applications. Llevado a cabo en Visakhapatnam, India. https://dx.doi.org/10.1007/978-81-322-2752-6_8

Heredia, Y. y García, B. (2013). Relaciones entre el desempeño académico y autorregulaciónautoestima- inteligencia emocional en estudiantes de secundaria. En M.C. Barrón Tirado, XII Congreso Nacional de Investigación Educativa. Llevado a cabo en Guanajuato, México. 
Kumar, M., Emory, J. y Chopella, V. (2018). Usability Analysis of Virtual Labs. En 18th International Conference on Advanced Learning Technologies. Llevado a cabo en Bombay, Mumbai India.

Pearson, C. y Kudzai, C. (2015). Virtual laboratories: a solution for tertiary science education in botswana? European Journal of Logistics, Purchasing and Supply Chain Management, 3(3), $12-24$.

Pérez Pinal, F., Nava, S., Núñez Pérez, J., Araujo Vargas, I., Vera Cárdenas, E. y Barranco Gutierrez, A. (2016). Experimental B-Learning Laboratory for an Electrical Machines Undergraduate Course. IEEE Latin America Transactions, 14(2), 524-529.

Pizzonia, M. y Rimondini, M. (2016). Netkit: Network emulation for education. Software-Practice \& Experience, 46(2), 133-165. NJ, USA: Wiley-Blackwell.

Potkonjak, V., Gardner, M., Callaghan, V., Mattila, P., Guetl, C., Petrovic, V.M. y Jovanovic, K. (2016). Virtual laboratories for education in science, technology, and engineering: A review. Computers \& Education, $95(\mathrm{~s} / \mathrm{n}), 309-327$.

Raessens, J. (2014). The Ludification of Culture. En M. Fuchs, S. Fizek, N. Schrape y P. Ruffino (eds.), Rethinking gamification. Lüneburg, Alemania: Meson Press.

Redel-Macías, M.D., Pinzi, S., Martínez-Jiménez, M.P., Dorado, G. y Dorado, M.P. (2016). Virtual laboratory on biomass for energy generation. Journal of Cleaner Production, 112(2016), 3842-3851. http://dx.doi.org/10.1016/j.jclepro.2015.07.075

Roux, R. y Anzures, E.E. (2014). Estrategias de aprendizaje y su relación con el rendimiento académico en estudiantes de una escuela privada de educación media superior. Revista Electrónica Actualidades Investigativas en Educación, 15(1). Recuperado de www.redalyc. org $/ \mathrm{html} / 447 / 44733027014 /$

Ruano, I., Gámez, J., Dormido, S. y Gómez, J. (2016). A methodology to obtain learning effective laboratories with learning management system integration. IEEE Transactions on Learning Technologies, 9(4), 391-399.

Sampieri, R., Fernández, C. y Baptista, P. (2014). Metodología de la investigación. México: McGraw-Hill.

Ushakov, Y., Shukhman, A., Polezhaev, P., Legashev, L. (2017). Virtual cloud network laboratory based on IaaS for university IT education. En C. Douligeris y M.E. Auer, IEEE Global Engineering Education Conference (EDUCON). Llevado a cabo en Atenas, Grecia.

Wuttke, H., Hamann, M. y Henke, K. (2015). Integration of Remote and Virtual Laboratories in the Educational Process. En M.E. Auer (ed.), 12th International Conference on Remote Engineering and Virtual Instrumentation (REV). Llevado a cabo en Bangkok, Thailand. 